\title{
Puesta en valor del fruto "Ushun" mediante la caracterización botánica, físicoquímica y fitoquímica, Cullaspampa, distrito de Caraz - Ancash
}

\author{
Deysi Mendo-Ponce¹, Gimena Lázaro², Jorge Leyva³ , Deysi Guzmán y Eloy \\ Cuellar ${ }^{5}$
}

Recibido: 20 octubre 2020 | Aceptado: 15 octubre 2021

\section{RESUMEN}

El presente estudio tuvo como objetivo generar información botánica, fisicoquímica y fitoquímica para poner en valor al "Ushun", especie frutal nativa del Perú, mediante evaluaciones físicas y fitoquímicas que servirán como base para determinar sus usos potenciales. Se colectaron muestras dendrológicas en la localidad de Cullaspampa en Caraz, tratándose de la especie Bunchosia armeniaca perteneciente a la familia Malpighiaceae. Las características físicas del fruto presentaron una media del tamaño longitudinal y diametral de 2,00 y 1,72 cm, respectivamente, y un peso promedio de $4,75 \mathrm{~g}$, la cáscara pesó en promedio 1,12 g, y constituye un $24,05 \%$ respecto al fruto; la pulpa, presentó una media de 1,94 $\mathrm{g}$, el rendimiento del despulpado fue de 40,30\%, la concentración de azúcar fue de $40^{\circ}$ Brix y 0,01\% expresado como ácido cítrico. En cuanto a las semillas, presentaron un tamaño longitudinal promedio de $1,32 \mathrm{~cm}$ y diámetro de $0,57 \mathrm{~cm}$, un peso promedio de $1,13 \mathrm{~g}$ representando el $24,28 \%$ del fruto. Estos resultados fueron obtenidos al 68,79 $\pm 1,45$ y $60,82 \pm 1,26 \%$ de humedad del fruto y de la pulpa, respectivamente. Asimismo, la marcha fitoquímica determinó que los fitoconstituyentes presentes fueron: alcaloides en general, aminas terciarias, fenoles, flavonoides, compuestos grasos y grupos funcionales. Se concluyó que posee propiedad antibacteriana, antimicrobiana, antiviral, antioxidante y antiinflamatoria; además de, valor terapéutico, como relajante muscular, tranquilizante, antitusivo y analgésico. Debido a sus características físicas y fitoquímicas, el "Ushun" cuenta con un elevado potencial para ser incluido en la industria.

Palabras clave: Bunchosia armeniaca, fitoconstituyentes, metabolitos secundarios.

\footnotetext{
${ }^{1}$ Universidad Nacional Agraria La Molina, deysi.mendo.p@gmail.com

${ }^{2}$ Universidad Nacional Agraria La Molina, gimena199805@gmail.com

${ }^{3}$ Universidad Nacional Agraria La Molina, jorge4antono@gmail.com

${ }^{4}$ Universidad Nacional Agraria La Molina, deysigl@lamolina.edu.pe

${ }^{5}$ Universidad Nacional Agraria La Molina, eloycuellar@lamolina.edu.pe
} 


\section{ABSTRACT}

The present study had the objective of generating taxonomic, physicochemical and phytochemical information to value the "Ushun", native fruit species of Peru, through physical and phytochemical evaluations that will serve as a basis to determine its potential uses. Dendrological samples were collected in the locality of Cullaspampa in Caraz, determining that the species was Bunchosia armeniaca belonging to the Malpighiaceae family. The physical characteristics of the fruit presented an average longitudinal and diametric size of 2,00 cm and 1,72 cm, respectively, and an average weight of $4,75 \mathrm{~g}$, the peel presented an average weight of $1,12 \mathrm{~g}$, and constitutes $24,05 \%$ regarding the fruit, the pulp, presented an average of $1,94 \mathrm{~g}$, the pulping yield was $40,30 \%$, its present sweetness is $40^{\circ}$ Brix and 0,01\% expressed as citric acid. As for the seeds, they presented an average longitudinal size of $1,32 \mathrm{~cm}$ and a diametric of $0,57 \mathrm{~cm}$, an average weight of $1,13 \mathrm{~g}$ representing $24,28 \%$ of the fruit. These data were obtained at a humidity percentage of the fruit and pulp of $68,79 \pm 1,45 \%$, and $60,82 \pm 1.26$, respectively. Also, the phytochemical screening determined that the phytoconstituents present were alkaloids in general, tertiary amines, phenols, flavonoids, fatty compounds and functional groups. It was concluded that it has antibacterial, antimicrobial, antiviral, antioxidant and anti-inflammatory properties, therapeutic value, as muscle relaxants, tranquilizer, antitussive and analgesic. Due to its physical and phytochemical characteristics, "Ushun" has a high potential to be included in the industry.

Keywords: Bunchosia armeniaca, phytoconstituents, secondary metabolites

\section{INTRODUCCIÓN}

El Perú es un país megadiverso, dentro de la inmensidad de su flora, cuenta con 623 especies de árboles frutales aproximadamente, de los cuales 523 son nativas (CONCYTEC, 2016). Es indispensable revalorar las especies nativas, puesto que sus frutos podrían constituir seguridad alimentaria y una fuente económica adicional para los pobladores locales del Perú (Obregón, 2020). Según Jacobsen et al. (2003), nuestras especies nativas tienen ventajas comparativas debido al sabor, aroma único, consistencia y fina textura.

Existen prácticas antiguas para mejorar las características de ciertas especies. Una de ellas es la domesticación. Esta es una actividad que se ha realizado de forma tradicional en los huertos familiares, principalmente en especies forestales con fines de obtención de frutos (Cornelius y Ugarte, 2010). En el caso del "Ushun" existe evidencia arqueológica que demuestra que este árbol frutal nativo fue utilizado en culturas peruanas donde predominó las actividades de manejo y producción en chacras familiares como Mochica y Guitarreros (Fernandez y Rodriguez, 2007).

Es necesario justificar, a base de estudios, la importancia de las especies nativas, así como, conocer las características químicas de los frutos, metabolitos secundarios, los cuales son importantes para la planta 
puesto que, actúan como atrayentes para los polinizadores y tóxicos para los predadores (García y Carril, 2011). En el caso del "Ushun", especie frutal de la región andina, se utiliza como fruto fresco, mermeladas, jaleas, confitados, entre otros (Jacobsen et al., 2003). Además, cuenta con una ventaja agroexportadora, ya que al ser nativa se encuentra adaptada a las distintas condiciones edafológicas y climáticas del país, por lo que no exige tanta atención técnica y elevados costos de producción a comparación de cultivos de frutales no nativos (Mostacero et al., 2017).

Por ello, la presente investigación tiene como objetivo generar información botánica, física y fitoquímica para valorizar al "Ushun”, especie forestal nativa poco estudiada, ubicada en Cullaspampa, Ancash, a partir de la identificación de la especie, el estudio del fruto a nivel descriptivo de sus características físicas y químicas, y la determinación de sus metabolitos secundarios. El conocer dichas características, servirá como base para proponer posibles usos potenciales y a partir de ello visualizar a futuro planes de domesticación e incluso mejoramiento genético (Mostacero et al., 2017).

\section{MATERIALES y METODOLOGÍA}

\section{Área de estudio}

El lugar de estudio fue la localidad de Cullaspampa, distrito de Caraz, provincia de Huaylas, región Ancash (Figura 1). Según Weatherspark (2016)
Caraz posee una temperatura de $10^{\circ} \mathrm{C}$ a $24^{\circ} \mathrm{C}$ y rara vez baja a menos de $7^{\circ} \mathrm{C}$ o sube a más de $26^{\circ} \mathrm{C}$; en la época lluviosa de octubre a abril la precipitación acumulada se encuentra entre 13 a 29 mm; la topografía en un radio de 3 kilómetros tiene grandes variaciones de altitud tiene variaciones enormes de altitud, con un cambio máximo de altitud de 895 metros y una altitud promedio sobre el nivel del mar de 2,391 metros. El área en un radio de 3 kilómetros está cubierta de arbustos (56\%), árboles (16\%), pradera (15\%) y tierra de cultivo (14\%), en un radio de 16 kilómetros, de arbustos (47 \%) y pradera (24\%) y en un radio de 80 kilómetros, de arbustos (34 \%) y pradera (22 \%). Además, los pobladores locales mencionan que estas zonas han sido manejadas por sistemas agroforestales multiestratos, sistemas silvopastoriles y huertos familiares, durante los últimos años.

\section{Colecta botánica}

Para la identificación de la especie se colectaron de 3 a 5 muestras dendrológicas de los 5 individuos encontrados en el área de estudio; 3 de ellas solo con hojas y 5 con flores y/o frutos. La colecta se realizó en el mes de enero, ya que se encontraban en floración y principios de fructificación. Se realizó un prensado en campo. Luego se preservó con alcohol al $80 \%$, para después ser secadas en un horno. Por último, se realizó el montaje y se cotejó con especímenes del Herbario (MOL). 


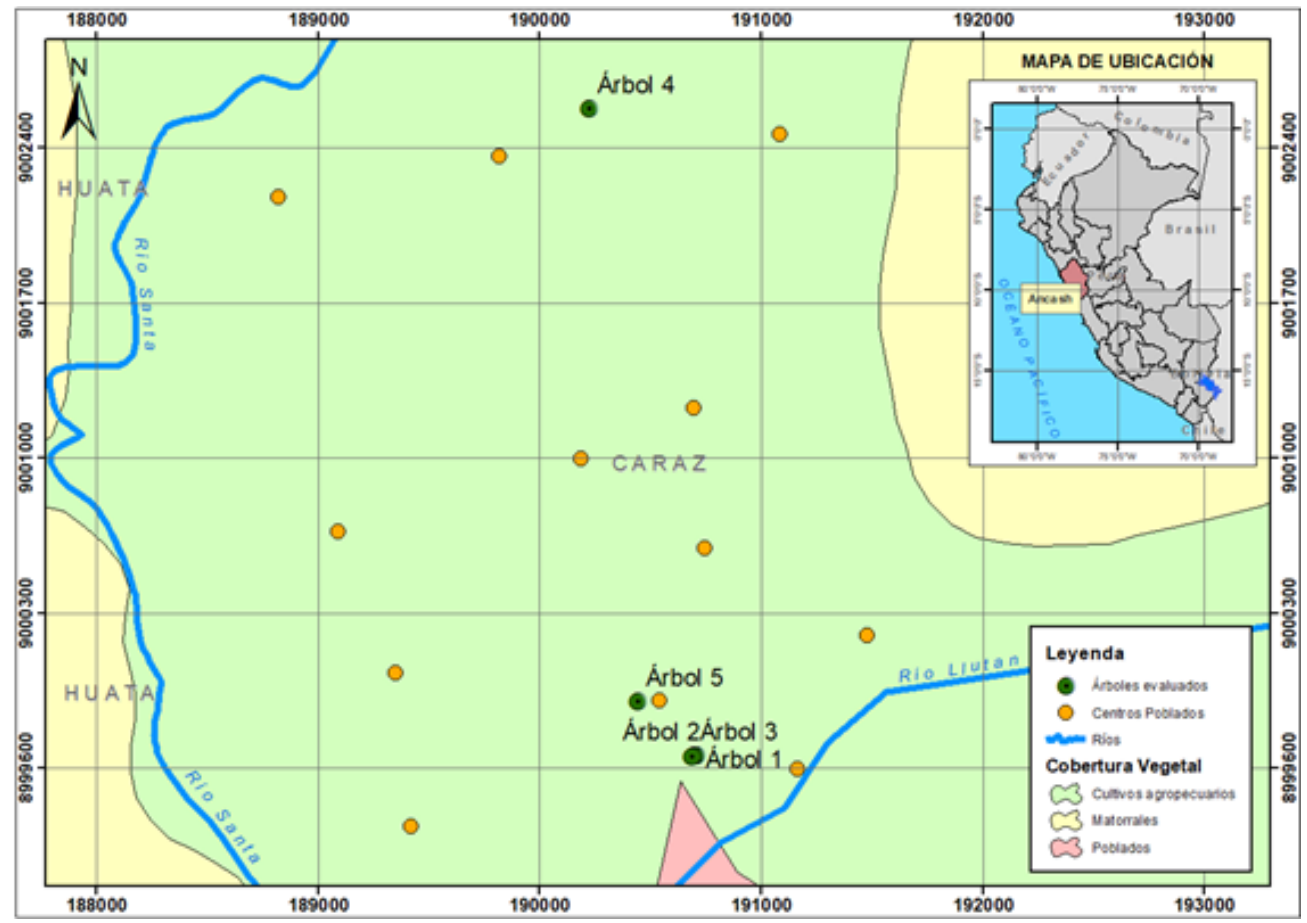

Figura 1.

Mapa del área de estudio con la ubicación de los árboles evaluados.

\section{Análisis de parámetros físicoquímicos}

Los equipos utilizados para los parámetros físicos, fueron: la balanza analítica de capacidad $200 \mathrm{~g}$, el vernier digital, el refractómetro, el colorímetro de Lovibond LC100 y la mufla. Las metodologías usadas para los análisis químicos y grados brix, fueron las establecidas por la Association of Official Analytical Chemistry (AOAC, 2005) $\mathrm{N}^{\circ} 942.15$ y $N^{\circ} 931.12$, respectivamente.

De los frutos maduros colectados se tomó una muestra al azar de 80 frutos, los cuales se utilizaron para las evaluaciones físicas y químicas, las que se desarrollan secuencialmente; se registró el peso para cada fruto, luego se realizaron las medidas biométricas. Posteriormente, se separaron pulpa, semillas y cáscara, se midió el rendimiento de pulpa y color de la misma. Se determinó el contenido de humedad del fruto entero y de la pulpa en laboratorio. En cuanto al color, se halló el índice del color (IC) a partir de los parámetros $L^{*}, a^{*}, b^{*}$, mediante la fórmula de $I C=(\operatorname{ax1000)} \div$ (Lxb). Donde; L*: luminosidad, $a^{*}$ : coordenadas rojo/verde, $b^{*}$ : coordenadas amarillo/azul.

\section{Análisis fitoquímicos}

Previo a realizar la marcha fitoquímica, se realizó el ensayo de solubilidad para determinar el solvente apropiado para identificar los fitoquímicos presentes en la pulpa del fruto. Para ello se utilizó etanol a diferentes concentraciones: $30 \%$, 50\%, 70\%, 96\% y agua, luego en cinco matraces 
diferentes se pesó 5 g de pulpa de humedad conocida y se le añadió 50 $\mathrm{mL}$ de cada concentración a cada matraz, los cuales se cubrieron con aluminio y se llevaron a agitación por una hora a $200 \mathrm{rpm}$, luego de ese tiempo se filtró; de los filtrados se determinaron los sólidos disueltos para determinar el porcentaje de solubilidad para cada concentración de solvente. Posteriormente, con solvente de mayor valor de solubilidad se realizó la marcha fitoquímica mediante la metodología propuesta por Guzmán (2018) para la identificación de metabolitos secundarios.

\section{Análisis estadísticos}

Se analizaron los parámetros físicos mediante estadísticos descriptivos, mientras que, la distribución de frecuencias del peso del fruto, tamaño longitudinal y diametral de fruto, peso de pulpa, rendimiento del despulpado, y peso de semillas, del fruto "Ushun" se realizó a través del análisis en el Software R (R Core Team, 2020).

\section{RESULTADOS Y DISCUSIONES}

\section{Descripción taxonómica}

La descripción dendrológica de los 5 individuos presentó las siguientes características: corteza externa lenticelada, hojas simples, opuestas y decusadas, con presencia de glándulas en el envés, inflorescencia axilar racemosa, flores con presencia de glándulas en la base del cáliz y frutos en racimo de tipo drupa. Little y Dixon (1969), señalan que las características más resaltantes y propias de la especie son las glándulas extraflorales. Dichas particularidades, en conjunto con la certificación del jefe del Laboratorio de Dendrología y Herbario (MOL) de la Universidad Nacional Agraria la Molina, el Ph.D. Carlos Reynel Rodríguez, determinaron que la especie estudiada es Bunchosia armeniaca (Cav.) DC de la familia Malpighiaceae.

\section{Evaluación de las características fisicoquímicas del fruto de "Ushun"}

Los resultados del análisis estadístico de las características físicas del fruto se detallan en la Tabla 1.

En promedio, la longitud y diámetro del fruto nativo fueron 2,00 y $1,72 \mathrm{~cm}$ respectivamente. En cuanto al primero, estos valores son menores a los obtenidos por Pérez (2017), quien realizó el estudio en la zona del distrito de Sayán de la provincia de Huaura, departamento de Lima y obtuvo valores de 3 a $4 \mathrm{~cm}$. Estas diferencias se pueden explicar debido a los distintos ecotipos de la especie, que exponen características particulares según la zona de estudio y condición de madurez (Pinedo, 2008). El rendimiento de pulpa fue de $40 \%$, la cual contenía $40^{\circ}$ Brix. Este último resultado difiere de lo obtenido por Karunasena et al. (2018), el cual fue de $21,33 \pm 0,57 \%$. Es decir, el fruto estudiado contiene una mayor cantidad de sólidos disueltos en la pulpa del fruto y refleja un mayor estado de madurez (Pinzón et al., 2007). Además, los factores externos como las condiciones climáticas del sitio en donde se desarrolla el fruto; así como, la variedad del fruto y rendimiento de asimilación de las hojas, influyen en la cantidad de azúcares (Osterloch et al., 1996, citado por Pinzón et al., 2007). 
El valor encontrado para la acidez de la pulpa fue 0,01\% expresado como ácido cítrico, el cual resultó menor a lo determinado por Karunasena et al. (2018) con $0.32 \pm 0.06 \%$. Esta diferencia se originó por la disminución de ácidos titulables por las condiciones de maduración del fruto estudiado, ya que los ácidos orgánicos son usados como sustratos respiratorios (Moreno y Deaquiz, 2016).

\begin{tabular}{lccccc}
\hline \multicolumn{1}{c}{ Variable } & Media & D.E. & CV & Máximo & Mínimo \\
\hline Peso de fruto (g) & 4,75 & 1,72 & 36,23 & 8,27 & 2,06 \\
Tamaño longitudinal fruto (cm) & 2,00 & 0,21 & 10,58 & 2,44 & 1,58 \\
Tamaño diametral fruto (cm) & 1,72 & 0,19 & 11,27 & 2,08 & 1,32 \\
Peso de pulpa (g) & 1,94 & 0,83 & 42,84 & 3,72 & 0,80 \\
Rendimiento de despulpado (\%) & 40,30 & 6,27 & 15,57 & 52,66 & 25,46 \\
Peso de cáscara (g) & 1,12 & 0,41 & 36,36 & 2,08 & 0,48 \\
Porcentaje de cáscara (\%) & 24,05 & 3,87 & 16,10 & 33,84 & 17,52 \\
Peso de semilla (g) & 1,13 & 0,44 & 38,69 & 2,21 & 0,68 \\
Porcentaje de semilla (\%) & 24,28 & 4,72 & 19,45 & 33,27 & 16,24 \\
Tamaño longitudinal semilla (cm) & 1,32 & 0,22 & 16,56 & 1,69 & 0,86 \\
Tamaño diametral semilla (cm) & 0,57 & 0,25 & 44,37 & 0,87 & 0,12 \\
\hline
\end{tabular}

Tabla 1.

Estadísticos descriptivos de las variables cuantitativas del fruto "Ushun"

La Figura 2 muestra que la distribución de frecuencias del peso del fruto, el tamaño longitudinal, y el rendimiento del despulpado, tienden a la normalidad, al igual que, las especies en proceso de domesticación (Pinedo, 2008). En el caso del peso de la semilla su gráfica de frecuencia tiende a tener una distribución marcada en una clase, lo cual se ve reflejado en frutos con características físicas similares.

En cuanto al color de la cáscara y pulpa del fruto de las muestras evaluadas, se obtuvo valores promedio para los parámetros $L^{*} 29,72 \pm 8,94, a^{*} 8,61 \pm 4,91$, b* $11,30 \pm 3,91, C^{*} 14,33 \pm 5,98$ y h*54,26 $\pm 7,49$ y L* $23 \pm 2,69, a^{*} 29,10 \pm 1,84, b^{*}$ $26,30 \pm 3,82, C^{*} 39,35 \pm 1,2$ y h* $42 \pm 5,94$, lo cual indica un color opaco entre rojo y amarillo, y anaranjado fuerte, respectivamente. Dichas tonalidades se verifican con los valores del índice de color de 25,63 en la cáscara y 42,09 en la pulpa, los cuales representan colores que van desde naranja intenso al rojo profundo (Vignoni et al., 2006). Sin embargo, las coordenadas obtenidas por Karunasena et al. (2018) no coinciden con los resultados expuestos, lo cual se ve reflejado en las variaciones de $L^{*}, a^{*}$ y $b^{*}$ del espacio de color CIE-L*a*b* (Castro et al., 2013), razón por la cual se deduce que ambos estudios se realizaron con frutos en distintas etapas de maduración, debido a la disminución de clorofila e incremento de carotenoides y 
(a) Peso del fruto

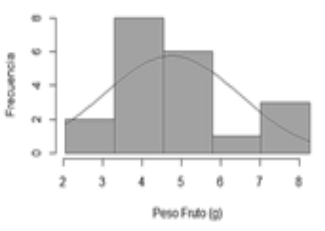

(c) Tamaño diametral del fruto

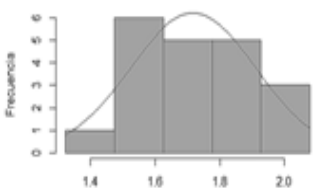

Dameso Findo (oni)

(e) Rendimiento al despulpeo

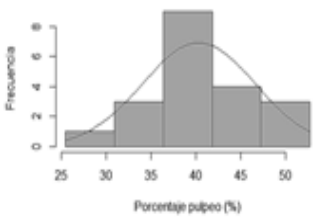

(b) Tamaño longitudinal del fruto

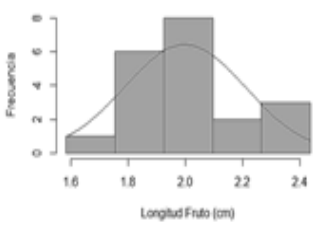

(d) Peso de la pulpa

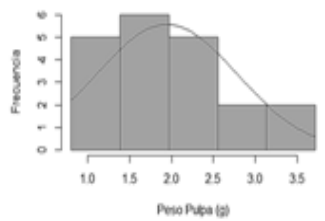

(f) Peso de la semilla

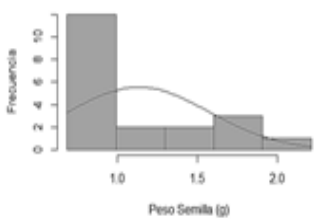

Figura 2. Distribución de frecuencias de los parámetros físicos: (a)peso de fruto, (b)tamaño longitudinal, (c)tamaño diametral del fruto, (d)peso de pulpa, (e)rendimiento del despulpado, y peso de semillas, del fruto "Ushun".

antocianinas según el estado del fruto (Agriculturers, 2017).

El porcentaje de humedad del fruto y pulpa fue en promedio $68,80 \pm 1,45$ y $60,82 \pm 1,26 \%$, respectivamente. En función a los resultados obtenidos, Mera (2015) indica que la alta cantidad de agua en el fruto incrementa el grado de las velocidades de las reacciones enzimáticas que se dan en el fruto. Además, según Herrera y Vela (2016) esto puede inducir el crecimiento microbiano, presencia de hongos y el deterioro.

Evaluación fitoquímica de la pulpa de "Ushun"

Se determinó que la pulpa de $B$. armeniaca es soluble en agua al igual que los resultados expuestos por Pérez (2017) y, Ocaña y Tadeo (2019). Esto indicó que existe mayor cantidad de componentes de alta polaridad (Ruíz, 2009).

La marcha fitoquímica en extracto acuoso obtuvo 7 reacciones positivas (Tabla 2). Se detectó presencia de alcaloides y aminas terciarias, mediante las reacciones observadas en los ensayos de Dragendorff, Wagner y Mayer (Canepa, 2018). La presencia de alcaloides podría conferirle algunas propiedades terapéuticas, como relajantes musculares (García y PérezUrria, 2009).

Por otro lado, la presencia de fenoles resultó positiva en la reacción con gelatina salada. Sin embargo, no se encontró taninos hidrolizables 0 pirogálicos debido al resultado negativo con la prueba de cloruro férrico.

Según Queiroz et al. (2015) B. armeniaca presenta flavonoides. Esto fue confirmado con la prueba de Shinoda, la cual determinó la presencia de isoflavanonas, chalconas y auronas. El autor señala que la importancia de estos fitoconstituyentes se debe a que generan una gran actividad antibacteriana, antimicrobiana, antiviral, antioxidante y antiinflamatoria.

La presencia de compuestos grasos resultó positiva, los cuales según Mendoza y Calvo (2010) son importantes en la participación de la regulación del metabolismo.

Además, se encontró presencia de grupos funcionales, los cuales confieren propiedades bioquímicas en algunas biomoléculas (Look, 1994). 


\begin{tabular}{|c|c|c|}
\hline Metabolito & Reactivo & Reacción \\
\hline & Dragendorff & Positivo \\
\hline & Wagner & Positivo \\
\hline & Mayer & Positivo \\
\hline \multirow[t]{3}{*}{ Alcaloides } & Erdman & Negativo \\
\hline & Marquis & Negativo \\
\hline & Otto & Negativo \\
\hline Compuestos grasos & Sudan III & Positivo \\
\hline Azúcares reductores & Fehling $A$ y fehling $B$ & Negativo \\
\hline Saponinas & Agua destilada & Negativo \\
\hline Anillos aromáticos & $\mathrm{H}_{2} \mathrm{SO} 4$ & Negativo \\
\hline \multirow{2}{*}{ Fenoles } & $\mathrm{FeCl}_{3}$ & Negativo \\
\hline & Gelatina salada & Positivo \\
\hline Flavonoides & Shinoda & Positivo \\
\hline Grupos funcionales & $\mathrm{KMnO}_{4}$ & Positivo \\
\hline Antraquinonas y/o naftoquinonas & Borntrager & Negativo \\
\hline $\begin{array}{l}\text { Glucósidos cardiacos, para anillos } \gamma- \\
\text { lactonas- } \alpha, \beta \text {-insaturados. }\end{array}$ & Kedde & Negativo \\
\hline Proteínas & Millon's & Negativo \\
\hline Núcleos esteroidales y triterpénicos & Ácido tricloroacético & Negativo \\
\hline Sapogeninas esteroidales y triterpenoidales & Rosenthaler & Negativo \\
\hline Sapogeninas & $\mathrm{SbCl}_{3}$ en $\mathrm{HClO}_{4}$ & Negativo \\
\hline
\end{tabular}

Tabla 2.

Resultado del ensayo fitoquímico en pulpa de B. armeniaca.

\section{CONCLUSIONES}

Los frutos estudiados pertenecen a la Además, La pulpa presenta alcaloides, especie nativa Bunchosia armeniaca, aminas terciarias, fenoles, flavonoides, perteneciente a la familia Malpighiaceae. compuestos grasos y grupos funcionales. Por lo que, el fruto del "Ushun" debido a

Según las distribuciones de las variables biométricas, los individuos evaluados presentan tendencias a la domesticación. incluido en la industria. 
En ese sentido, se recomienda realizar investigaciones como el análisis proximal del fruto para la $R$ determinación de su composición nutricional, así como abordar la parte silvicultural mediante la evaluación de métodos de propagación del fruto a nivel de vivero.

\section{AGRADECIMIENTO}

A la empresa Laboratorios Hersil S.A. y su afiliación a la Universidad Nacional Agraria la Molina por el financiamiento de subvención para la realización del proyecto. Al laboratorio Procesos de Transformación Química de los Productos Forestales Maderables y al Herbario MOL, ambas instalaciones de la Facultad de Ciencias Forestales UNALM, por la disposición de sus instalaciones y equipos. Al PhD. Carlos Reynel Rodriguez, a Gianlucca Monteverde, a Juan Julca Torres, a Erick Valerio, a José Coronado y a los miembros del Círculo de Investigación de Plantaciones Forestales (CIPLAN Forestal) por su apoyo en el presente estudio.

A los pobladores del distrito de Caraz por compartir sus conocimientos y hospitalidad para el desarrollo de la fase de campo.

\section{LITERATURA CITADA}

Agriculturers. (15 de junio del 2017). Maduración del fruto y términos de uso común en post cosecha. https:// bit.ly/33wGTFP

AOAC. Association of Official Analytical Chemistry. (2005). Official Methods of Analysis. Patricia Cunniff. Virginia. USA.
Canepa, F. (2018). Evaluación Química del Fruto de "Charán" (Caesalpinia paipái Ruis \& Pavón), proveniente de Motupe, Lambayeque [Tesis de ingeniero, Universidad Nacional Agraria la Molina]. https://bit.ly/2Alfnig

Castro, J., Cerquera, N. y Gutierres, G. (2013). Determinación del color del exocarpio como indicador de desarrollo fisiológico y madurez en La guayaba pera (Psidium guajava cv. Guayaba pera), utilizando técnicas de procesamiento digital de imágenes. Revista EIA, 10(19), 79-89. https://bit.ly/2Sr7q0X

CONCYTEC. (2016). Programa Nacional Transversal de Valorización de la Biodiversidad. https://bit.ly/36CC8um

Cornelius, J. y Ugarte-Guerra, L. (2010). Introducción a la genética y domesticación forestal para la agroforestería y silvicultura. Lima, Perú. Centro mundial para la agroforestería (ICRAF). https:// bit.ly/2TM3mJx

Fernández, A. y Rodríguez, E. (2007). Etnobotánica del Perú Pre-hispano. Ediciones Herbarium Truxillense (HUT). Universidad Nacional de Trujillo. https:// bit.ly/2B5j4ZQ

García, A. Á. y Carril, E. P. (2011). Metabolismo secundario de plantas. Reduca (biología), 2(3), 119-145. https:// bit.ly/36JtHxs

García, A. y Pérez-Urria, E. (2009). Metabolismo secundario de plantas. Reduca (Biología), Serie Fisiología Vegetal, 2(3), 119-145. https:// bit.ly/3cgPloc 
Guzmán, D. (2018). Guía de prácticas de química forestal. Departamento de Industrias Forestales, Universidad Nacional Agraria la Molina.

Herrera M. y Vela N. (2016). Caracterización fitoquímica y parámetros fisicoquímicos de hoja, corteza y raíz de Unonopsis floribunda Diels (ICOJA). [Tesis de químico farmacéutico, Universidad Nacional de la Amazonía Peruana]. https:// bit.ly/3dg52mr

Jacobsen, S. E., Mujica, A. y Ortiz, R. (2003). La importancia de los cultivos andinos. Fermentum. Revista Venezolana de Sociología Y Antropología, 13(36), 14-24. https:// bit.ly/3gwUSjt

Karunasena, G., Chandrajith, V. y Nawaratne, S. (2018). Physicochemical characteristics of peanut butter fruit (Bunchosia armeniaca). International Journal of Food Science and Nutrition, 3(3), 46-51. https://bit.ly/2SriA5N

Little, E. L. y Dixon, R.G. (1969). Árboles comunes de la Provincia de Esmeraldas, Ecuador. Cuerpo de Paz. https: / / bit.ly/2M8HzHz

Look, O. (1994). Investigación Fitoquímica. Métodos en el estudio de productos naturales, Perú. Pontificia Universidad Católica del Perú. https:// bit.ly/2XeSiHi

Mendoza, E., y Calvo, M. D. (2010). Bromatología: composición y propiedades de los alimentos. Mc Graw-Hill.
Mera Paredes, S. J. (2015). Manejo poscosecha de frutas y hortalizas [Tesis de ingeniero, Universidad Nacional de la Amazonía Peruana]. https:// bit.ly/2TPWyL1

Moreno, B. y Deaquiz, Y. (2016). Caracterización de parámetros fisicoquímicos en frutos de mora (Rubus alpinus Macfad). Agroindustria y Ciencia de los Alimentos, 65(2), 130-136. http:// dx.doi.org/10.15446/acag.v65n2.45587

Mostacero León, J., Mejía Coico, F., Gastañadui Rosas, D., y De La Cruz Castillo, J. (2017). Inventario taxonómico, fitogeográfico y etnobotánico de frutales nativos del norte del Perú. Scientia Agropecuaria, 8(3), 215-224. https:// doi.org/10.17268/sci.agropecu.2017.03.04

Obregón La Rosa, A. J. (2020). Componentes de frutos nativos como fuente potencial de nutrientes en el requerimiento nutricional óptimo de grupos vulnerables. [Tesis de doctorado, Universidad Nacional Federico Villarreal]. https://bit.ly/3cf0aws

Ocaña J., Tadeo M. (2019). Evaluación farmacognóstica y cuantificación de fenoles total del fruto de Bunchosia armeniaca (Cav.) DC "Ciruela cansa boca". [Tesis de farmacia y bioquímica, Universidad Nacional de Trujillo]. https:// bit.ly/2Xa2G2R

Pérez, V . (2017). Estructura Química De Algunos Componentes Del Extracto Etanólico Del Fruto Bunchosia Armeniaca (Cansa Boca) Con Actividad Antioxidante Y Antimicrobiana. [Tesis de químico farmacéutico y bioquímico, Universidad Inca Garcilaso De La Vega]. https:// bit.ly/2Ai4kXm 
Pinedo, H., (2008). Caracterización de la variación geográfica-fenotípica de Mauritia flexuosa Lf (aguaje) en 03 sectores de la región Madre de DiosPerú. https://bit.ly/3dhJ0je

Pinzón, I., Fischner, G. y Corredor G. (2007). Determinación de los estados de madurez del fruto de la gulupa (Passiflora edulis Sims). Agronomía Colombiana, 25(1), 83-95. https:// bit.ly/3blvNlb

Queiroz, G., Heller M., Arruda-Silva F., Nascimento M., Micke G., Dalmarco M., Pizzolatti M. y Brighente, I. (2015). Antibacterial and Anti-Inflammatory activities of Bunchosia armeniaca (Cav.) DC. (Malpighiaceae). Rec. Nat. Prod, 9(3), 419-431. https:// bit.ly/3ddJSVT

R Core Team. (2020). R: A Language and environment for statistical computing Foundation for statistical computing. Vienna, Austria. www.Rproyect.org/

Ruíz, S. (2009). Contribución del estudio farmacognóstico y farmacodinámico de las hojas de Mangifera indica $L$. al uso medicinal. [Tesis de doctor, Universidad Nacional de Trujillo]. https://bit.ly/2TOxhAR

Vignoni, A., Césari M., Forte, M., y Mirábile, L. (2006). Determinación de índice de color en ajo picado. Información tecnológica, 17(6), 63-67. http://dx.doi.org/10.4067/

S0718-07642006000600011
Weathersparck (2016). Clima promedio en Carás. https://es.weatherspark.com/ y/20520/Clima-promedio-en-Car\%C3\%A1sPer\%C3\%BA-durante-todo-el-a\%C3\%B1o parámetros 